\section{Factores de riesgo asociados a síntomas depresivos post parto en mujeres de bajo riesgo obstétrico atendidas en el sistema público}

\author{
ANGELINA DOIS $^{1, \mathrm{a}}$, CLAUDIA URIBE $^{1, \mathrm{~b}}$, \\ LUIS VILLARROEL ${ }^{2, \mathrm{c}}$, AIXA CONTRERAS ${ }^{1, \mathrm{~d}}$
}

\section{Risk factors for post partum depression}

Background: Postpartum depression (PPD) is a public health problem with high prevalence in Chile. Many factors are associated with PPD. Aim: To analyze the factors associated with the incidence of depressive symptoms (SD) in women with low obstetric risk. Material and Methods: Cross-sectional analytical study on a sample of 105 postpartum women with low obstetric risk assessed by the Edinburgh Depression Scale at the eighth week postpartum. Results: A 37\% prevalence of depressive symptoms was found. Univariate analysis showed that the perception of family functioning, overcrowding and number of siblings, were significantly associated with postpartum depressive symptoms. A multiple regression model only accepted family functioning as a predictor of depression. Conclusions: Perception of family functioning was the only variable that explained in part the presence of depressive symptoms in women with low obstetric risk.

(Rev Med Chile 2012; 140: 719-725).

Key words: Depression; Life style; Low obstetric risk; Post partum period.
'Escuela de Enfermería, Facultad de Medicina, Pontificia Universidad Católica de Chile, Santiago, Chile. ${ }^{2}$ Departamento de

Salud Pública, Facultad

de Medicina, Pontificia

Universidad Católica de

Chile, Santiago, Chile.

aEnfermera-matrona,

Magíster en Psicología de

la Salud.

bEnfermera-matrona, Magíster en Humanización de

la Salud, Directora Proyecto FONDEF D07i1046.

'Estadístico, Doctor (PhD)

en Estadística.

'Enfermera-matrona,

Magíster en Psicología Social Comunitaria.

Financiamiento: Proyecto FONDEF D07i1046. CONICYT, Pontificia

Universidad Católica de Chile y Servicio de Salud

Metropolitano Sur Oriente.

Recibido el 28 de septiembre de 2011, aceptado el 19 de enero de 2012.

Correspondencia a:

E.M. MS Angelina Dois, Escuela de Enfermería, Pontificia Universidad Católica de Chile. Vicuña Mackenna 4860, Macul, Santiago

Fono: $56-2-3545838$

Fax: 56-2-3547025

E-mail: adois@uc.cl

$$
\text { L }
$$
a depresión postparto (DPP) es el trastorno del ánimo de mayor prevalencia asociado $\checkmark$ al nacimiento, su sintomatología aparece entre las 4 y 30 semanas del puerperio y se caracteriza por la presencia de síntomas incapacitantes de incomodidad y afrontamiento, que pueden verse exacerbados por baja autoestima materna, falta de confianza y expectativas irreales sobre la maternidad $^{1-3}$. Se calcula que afecta a 19,2\% de las puérperas durante los tres primeros meses postparto ${ }^{4}$, pero su prevalencia puede variar entre $3 \%$ y $30 \%$ durante el primer año dependiendo del método de medición y del tiempo transcurrido desde el nacimiento ${ }^{1,3}$. Durante los primeros tres meses postparto ocurre entre $40 \%$ y $70 \%$ de los casos, con una recidiva de 1:3 a 1:4 si la madre no recibe el tratamiento correcto 5 . Aun así, hay una proporción de ellos que no mejora pudiendo incluso seguir un curso crónico ${ }^{3}$.
Los efectos que este cuadro tiene en la relación madre-hijo(a) son universales, su impacto en el desarrollo del hijo(a) está directamente relacionado con la duración e intensidad del mismo ${ }^{2}$ asociándose al establecimiento de apegos inseguros, deterioro del desarrollo psicomotor del hijo(a) y cese precoz de la lactancia materna, entre otros ${ }^{1,6-8}$.

El diagnóstico de DPP confiere algunas dificultades ya que su sintomatología puede confundirse con comportamientos asociados a la adaptación normal al puerperio. Esto se ve reflejado en que alrededor de $50 \%$ de los casos de DPP no se diagnostica ni se trata oportunamente ${ }^{5}$ y por otro lado, cerca de la mitad de los cuadros depresivos diagnosticados durante el puerperio se iniciaron o continuaron su curso durante el embarazo ${ }^{9}$, aun cuando se conoce que el diagnóstico y tratamiento correcto previo al parto reduce la probabilidad de cursar una depresión en el período postnatal ${ }^{10}$. 
Cabe destacar que en Chile existe una política pública que garantiza la cobertura de atención de las personas con cuadros depresivos ${ }^{5}$.

Pese a que se han descrito una serie de factores asociados, no es posible distinguir una causa única que explique la ocurrencia de DPP, por lo que se sostiene su etiología multifactorial. Diversos estudios han encontrado una mayor asociación de DPP en mujeres con antecedentes personales de baja autoestima, trastornos de la personalidad, depresión y depresión postparto previas, dificultades en las relaciones maritales, redes de apoyo insuficientes o pobres, antecedentes de eventos estresantes y en particular, cuadros ansiosos, depresivos y de estrés durante el embarazo ${ }^{3,4,10-12}$. También se ha descrito una asociación discreta con la presencia de tristeza postparto severa, la experiencia el parto, las complicaciones obstétricas y las características de la salud y temperamento del recién nacido ${ }^{3,13,14}$. Sin embargo, no hay estudios que demuestren la asociación de diferentes variables en mujeres consideradas de mínimo riesgo. Por esta razón, el objetivo de este trabajo es describir los factores asociados a la incidencia de síntomas depresivos (SD) en mujeres con embarazos de bajo riesgo obstétrico que se atendieron en centros de salud primaria del área suroriente de la Región Metropolitana. Los datos aquí reportados corresponden a la información basal obtenida durante la realización de un ensayo clínico aleatorio para la asistencia integral del parto en el servicio público (Proyecto FONDEF D07i1046).

\section{Material y Método}

Estudio analítico de corte transversal que evaluó sintomatología depresiva del post parto y su asociación con diversas variables biosociodemográficas de las participantes.

\section{Selección y tamaño de la muestra}

El universo comprendió a la totalidad de las mujeres con embarazos de bajo riesgo provenientes de seis centros de salud del área suroriente que asistieron su parto en una maternidad de Santiago durante el período julio de 2009-enero de 2010. Se seleccionó una muestra no aleatoria de $306 \mathrm{mu}-$ jeres puérperas mayores de 15 años que cursaron embarazos de bajo riesgo, sin diagnóstico previo de un trastorno del ánimo ni otra enfermedad psiquiátrica (lo que no descarta su existencia), que dieron a luz a recién nacidos únicos, sanos y de término y que estuvieron dispuestas a participar voluntariamente.

\section{Recolección de datos}

Los datos fueron recolectados por encuestadores capacitados, previa firma de Consentimiento Informado. El Proyecto de Investigación y el Consentimiento Informado fueron aprobados por el Comité de Ética de la Escuela de Enfermería de la Pontificia Universidad Católica de Chile y del Servicio de Salud Metropolitano Sur Oriente.

Durante el puerperio inmediato se aplicó un cuestionario para recoger información sobre antecedentes biosociodemográficos de las puérperas y la Guía de Síntomas Depresivos del MINSAL ${ }^{5}$. Posteriormente, se realizó un seguimiento telefónico alrededor de las 8 semanas postparto pesquisando síntomas depresivos a través de la Escala de Depresión de Edimburgo (EDE). Este período coincide con el de máxima prevalencia de la depresión posparto y con la disminución de los síntomas atribuibles a un período adaptativo normal ${ }^{7}$.

La EDE es un instrumento utilizado y validado en distintos países, incluyendo Chile ${ }^{15}$, con propósitos de tamizaje. Fue diseñado especialmente para reconocer síntomas anímicos en mujeres puérperas, posee una consistencia interna adecuada, $100 \%$ de sensibilidad y $80 \%$ de especificidad. Sus ítems se agrupan básicamente en torno a dos factores: tristeza y ansiedad, consta de 10 preguntas con cuatro opciones de respuesta cada una y puntajes que varían de 0 a 3 puntos, según la severidad de los síntomas, con una mayor sensibilidad con un punto de corte entre 9 y 10 puntos $^{7,16}$.

Las puérperas que obtuvieron puntajes sugerentes de DPP fueron derivadas a los Centros de Salud donde se controlaban para su evaluación en profundidad.

\section{Análisis de datos}

Los resultados de variables categóricas se presentan como número de casos y porcentaje y los de variables numéricas se presentan como medidas de tendencia central y dispersión. Se usó test $\mathrm{t}$ de Student para muestras independientes para comparación de promedios y test $\chi^{2}$ para comparación de porcentajes. La asociación lineal entre variables numéricas se determinó mediante 
correlación muestral de Pearson o correlación por rangos de Spearman, según si las variables involucradas en la asociación tenían o no origen ordinal. Finalmente, se usó regresión lineal para determinar la asociación conjunta de dos o más variables con el puntaje de depresión.

Se consideró significativo todo valor $p \leq 0,05$. Los análisis se hicieron usando el programa estadístico SAS 9.1 para Windows.

\section{Resultados}

Se encuestaron 306 usuarias durante el puerperio inmediato, de ellas, 201 tenían factores de riesgo sugerentes de trastornos del ánimo, equivalentes a 65,7\% del total, por lo que fueron derivadas a sus Centros de Salud y no se incluyeron en el seguimiento de este estudio. Las restantes 105 mujeres conformaron el grupo de seguimiento a las 8 semanas postparto.

Las puérperas estudiadas tuvieron una edad promedio de 24,5 años, con un rango entre 17 y 42 años. El 69,2\% tenía escolaridad media completa y en su mayoría se desempeñaban como dueñas de casa (55,2\%). Al momento del parto, 63,8\% del total mantenía una relación de convivencia estable con sus parejas (Tabla 1).

Del total de la muestra, 55,5\% no planificó su embarazo y $62,1 \%$ no usó ningún método contraceptivo. En relación a los antecedentes obstétricos, 46 eran primíparas (44,2\%), de acuerdo al tipo de parto, $91,4 \%$ fue vaginal (58,3\% sin episiotomía), $6,7 \%$ fórceps y 1,9\% cesárea. La edad gestacional promedio fue de 39,5 sem, el peso promedio de los recién nacidos fue de $3.362 \mathrm{~g}$, con un rango entre $2.480 \mathrm{~g} \mathrm{y}$ $4.280 \mathrm{~g}$ y el puntaje promedio de APGAR al minuto y 5 minutos fue de 9 (Tabla 2).

La Escala de Depresión de Edimburgo, medida a las 8 semanas del postparto, mostró una incidencia de SD en este grupo de $37,1 \%$. El 62,9\% de las encuestadas tuvo menos de 9 puntos, lo que las clasifica según el EDE como "sin riesgo de depresión". Del 37,1\% restante, 53,8\% tuvo entre 13 y 30 puntos lo que las clasifica dentro de la categoría "probable depresión”. Como puede observarse en la Tabla 3, en la evaluación por ítems de la EDE, se encontró que en relación a la disforia, las primigestas mostraron una tendencia a obtener una mayor cantidad de puntajes 0 en los ítems 1 y 2, no así en las multíparas en que la concentración de puntajes tendió a distribuirse entre 0 y 1 punto. Sólo el ítem 7 mostró una distribución más homogénea en ambos grupos. En relación al indicador de ansiedad, se observa una distribución homogénea de los grupos, sin embargo, en el ítem 4 existe una tendencia a concentrar las puntuaciones de ambos grupos en un puntaje 2, lo que da cuenta de niveles elevados de ansiedad materna. En el indicador de sentimientos de culpa y dificultades de concentración, si bien se observa una distribución homogénea de puntajes, en el grupo de

Tabla 1. Descripción de variables sociodemográficas

\begin{tabular}{|c|c|c|c|c|}
\hline \multicolumn{5}{|c|}{ Descripción de variables psicosociales categóricas } \\
\hline \multicolumn{2}{|l|}{ Variable } & \multicolumn{2}{|c|}{ Frecuencia } & rcentaje \\
\hline \multicolumn{2}{|l|}{$\begin{array}{ll}\text { Estado civil: } \\
- & \text { Casada } \\
- & \text { Convive } \\
- & \text { Soltera }\end{array}$} & $\begin{array}{l}26 \\
41 \\
38\end{array}$ & & $\begin{array}{l}24,76 \\
39,05 \\
36,19\end{array}$ \\
\hline \multicolumn{2}{|c|}{$\begin{array}{ll}- & \text { Fonasa A } \\
\text { - } & \text { Fonasa B } \\
\text { - } & \text { Fonasa C } \\
\text { - } & \text { Fonasa D } \\
\text { (*) } & \text { valor ausente: } 1\end{array}$} & $\begin{array}{r}56 \\
22 \\
18 \\
8\end{array}$ & & $\begin{array}{r}53,85 \\
21,15 \\
17,31 \\
7,69\end{array}$ \\
\hline \multicolumn{2}{|c|}{$\begin{array}{ll}\text { - } & \text { Básica completa } \\
\text { - } & \text { Media incompleta } \\
\text { - } & \text { Media completa } \\
\text { - } & \text { Universitaria incompleta } \\
\text { - } & \text { Universitaria completa } \\
\text { (*) } & \text { valor ausente: } 1\end{array}$} & $\begin{array}{r}12 \\
15 \\
72 \\
4 \\
1\end{array}$ & & $\begin{array}{r}11,54 \\
14,42 \\
69,23 \\
3,85 \\
0,96\end{array}$ \\
\hline \multicolumn{2}{|c|}{$\begin{array}{ll}\text { - } & \text { Sin actividad } \\
\text { - } & \text { Dueña de casa } \\
\text { - } & \text { Estudiante } \\
\text { - } & \text { Trab. Esporádico } \\
\text { - } & \text { Media jornada } \\
\text { - } & \text { Jornada completa }\end{array}$} & $\begin{array}{r}9 \\
58 \\
5 \\
2 \\
10 \\
21\end{array}$ & & $\begin{array}{c}8,57 \\
55,24 \\
4,76 \\
1,9 \\
9,52 \\
20\end{array}$ \\
\hline \multicolumn{5}{|c|}{ Descripción de variables psicosociales numéricas } \\
\hline Variable & Media & $\begin{array}{l}\text { Desviación } \\
\text { estándar }\end{array}$ & Mínimo & Máximo \\
\hline APGAR familiar & 8,9 & 1,27 & 4 & 10 \\
\hline Hacinamiento & 2,0 & 0,68 & 0,7 & 4 \\
\hline Promiscuidad & 1,48 & 0,37 & 0,6 & 2,5 \\
\hline
\end{tabular}


las multíparas hay una mayor concentración de puntajes 0 . Por último, en función del indicador de ideación suicida, éste se muestra con puntajes muy bajos en ambos grupos, lo que hace suponer que el suicidio no era una alternativa posible para ellas al momento de la encuesta.

Respecto de la asociación con algunas variables psicosociales (Tabla 4), se evaluó la relación entre

Tabla 2. Descripción de variables perinatales

\begin{tabular}{|c|c|c|c|c|c|c|c|}
\hline \multicolumn{3}{|c|}{ Descripción de variables perinatales categóricas } & \multicolumn{5}{|c|}{ Descripción de variables numéricas perinatales } \\
\hline $\begin{array}{l}\text { Variable } \\
\text { Embarazo planificado(*) }\end{array}$ & Frecuencia & Porcentaje & Variable & Media & $\begin{array}{c}\text { Desviación } \\
\text { estándar }\end{array}$ & $\begin{array}{l}\text { Míni- } \\
\text { mo }\end{array}$ & $\begin{array}{c}\text { Máxi- } \\
\text { mo }\end{array}$ \\
\hline \multirow{2}{*}{$\begin{array}{l}\text { Embarazo planificado(*) } \\
-\quad \text { Sí } \\
-\quad \text { No } \\
\text { (*) }^{*} \text { valor ausente: } 5\end{array}$} & \multirow{2}{*}{$\begin{array}{l}47 \\
53\end{array}$} & \multirow{2}{*}{$\begin{array}{l}44,76 \\
55,24\end{array}$} & Edad & 24,5 & 5,8 & 17 & 42 \\
\hline & & & Talla materna & 158,2 & 6,1 & 144 & 170 \\
\hline \multirow{5}{*}{$\begin{array}{l}\text { Uso método de planifica- } \\
\text { ción familiar }\left(^{*}\right) \\
-\quad \text { No } \\
-\quad \text { Natural } \\
-\quad \text { DIU } \\
-\quad \text { Hormonal } \\
-\quad \text { Otro } \\
\left.{ }^{*}\right) \text { valor ausente:2 }\end{array}$} & \multirow{5}{*}{$\begin{array}{r}64 \\
1 \\
6 \\
29 \\
3\end{array}$} & \multirow{5}{*}{$\begin{array}{r}62,14 \\
0,97 \\
5,83 \\
28,16 \\
2,91\end{array}$} & Hijos vivos & 0,76 & 0,93 & 0 & 5 \\
\hline & & & $\mathrm{N}^{\circ}$ de partos & 0,77 & 0,93 & 0 & 5 \\
\hline & & & $\begin{array}{l}\text { Edad gestacional } \\
\text { al parto }\end{array}$ & 39,5 & 0,99 & 37 & 41 \\
\hline & & & Peso RN & 3.362 & 372,5 & 2.480 & 4.280 \\
\hline & & & Talla RN & 49,3 & 3,6 & 34 & 55 \\
\hline \multirow{3}{*}{ 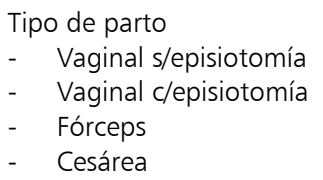 } & \multirow{3}{*}{$\begin{array}{r}56 \\
40 \\
7 \\
2\end{array}$} & \multirow{3}{*}{$\begin{array}{l}53,33 \\
38,1 \\
6,67 \\
1,9\end{array}$} & APGAR $1 \mathrm{~min}$ & 8,7 & 0,72 & 4 & 9 \\
\hline & & & APGAR $5 \mathrm{~min}$ & 9 & 0,20 & 8 & 10 \\
\hline & & & $\begin{array}{l}\text { Peso RN a las } \\
48 \text { hrs }\end{array}$ & 3.169 & 352,2 & 2.350 & 4.100 \\
\hline
\end{tabular}

Tabla 3. Análisis descriptivo escala de Edimburgo según grupo y puntaje

\begin{tabular}{|c|c|c|c|c|c|c|c|c|c|}
\hline \multirow[t]{3}{*}{ Síntoma } & \multirow[t]{3}{*}{ Ítem Escala de Edimburgo } & \multicolumn{8}{|c|}{$\begin{array}{l}\text { Distribución de frecuencia del ítem } \\
\text { según puntaje alcanzado }\end{array}$} \\
\hline & & \multicolumn{4}{|c|}{ Primigestas } & \multicolumn{4}{|c|}{ Multíparas } \\
\hline & & $\mathbf{0}$ & 1 & 2 & 3 & $\mathbf{0}$ & 1 & 2 & 3 \\
\hline \multirow[t]{5}{*}{ Disforia } & $\begin{array}{l}\text { Ítem 1: He sido capaz de reírme y ver el lado } \\
\text { positivo de las cosas }\end{array}$ & 38 & 6 & 2 & 0 & 35 & 18 & 3 & 1 \\
\hline & Ítem 2: He disfrutado mirar hacia adelante & 39 & 7 & 0 & 0 & 36 & 19 & 0 & 2 \\
\hline & $\begin{array}{l}\text { Ítem 7: Me siento tan desdichada que he } \\
\text { tenido dificultades para dormir }\end{array}$ & 18 & 12 & 15 & 1 & 20 & 15 & 14 & 8 \\
\hline & Ítem 8: Me siento triste o desgraciada & 22 & 20 & 2 & 2 & 23 & 21 & 12 & 1 \\
\hline & $\begin{array}{l}\text { Ítem 9: Me he sentido tan desdichada que he } \\
\text { estado llorando }\end{array}$ & 22 & 22 & 2 & 0 & 24 & 25 & 5 & 3 \\
\hline \multirow[t]{2}{*}{ Ansiedad } & $\begin{array}{l}\text { Ítem 4: He estado nerviosa o inquieta sin } \\
\text { tener motivo }\end{array}$ & 17 & 6 & 20 & 3 & 13 & 12 & 25 & 7 \\
\hline & $\begin{array}{l}\text { Ítem 5: He sentido miedo o he estado asusta- } \\
\text { diza sin tener motivo }\end{array}$ & 22 & 8 & 15 & 1 & 23 & 11 & 19 & 4 \\
\hline Sentimiento de culpa & $\begin{array}{l}\text { Ítem 3: Cuando las cosas han salido mal, me } \\
\text { he culpado a mi misma innecesariamente }\end{array}$ & 8 & 16 & 16 & 6 & 17 & 11 & 25 & 4 \\
\hline Dificultad de concentración & Ítem 6: Las cosas me han estado abrumando & 8 & 18 & 19 & 1 & 10 & 19 & 25 & 2 \\
\hline Ideación suicida & $\begin{array}{l}\text { Ítem 10: Se me ha ocurrido la idea de hacer- } \\
\text { me daño }\end{array}$ & 45 & 1 & 0 & 0 & 51 & 1 & 5 & 0 \\
\hline
\end{tabular}


ARTÍCULOS DE INVESTIGACIÓN

Predictores de síntomas depresivos post parto en mujeres de bajo riesgo obstétrico - A. Dois et al

Tabla 4. Correlación entre el puntaje EDE y variables biopsicosociales

\begin{tabular}{|c|c|c|}
\hline & \multicolumn{2}{|c|}{$\begin{array}{c}\text { Correlación con } \\
\text { puntaje EDE }\end{array}$} \\
\hline & $\begin{array}{l}\text { Correlación } \\
\text { de Spearman }\end{array}$ & Valor $p$ \\
\hline Edad materna & 0,18 & 0,23 \\
\hline Número de partos & 0,21 & 0,033 \\
\hline EG al parto & $-0,006$ & 0,95 \\
\hline APGAR familiar & $-0,38$ & $<0,001$ \\
\hline Hacinamiento & 0,22 & 0,02 \\
\hline Promiscuidad & 0,13 & 0,16 \\
\hline
\end{tabular}

Tabla 5. APGAR Familiar

\begin{tabular}{|c|c|c|c|}
\hline Preguntas & $\begin{array}{l}\text { Casi } \\
\text { siempre }\end{array}$ & $\begin{array}{c}\text { A } \\
\text { veces }\end{array}$ & $\begin{array}{c}\text { Casi } \\
\text { nunca }\end{array}$ \\
\hline \multicolumn{4}{|l|}{$\begin{array}{l}\text { Estoy satisfecho con la ayuda que recibo de } \\
\text { mi familia cuando algo me perturba }\end{array}$} \\
\hline \multicolumn{4}{|l|}{$\begin{array}{l}\text { Estoy satisfecho con la forma en que mi fa- } \\
\text { milia discute áreas de interés común y com- } \\
\text { parte los problemas resolviéndolos conmigo }\end{array}$} \\
\hline \multicolumn{4}{|l|}{$\begin{array}{l}\text { Encuentro que mi familia acepta mis deseos } \\
\text { de hacer nuevas actividades o cambios en } \\
\text { mi estilo de vida }\end{array}$} \\
\hline \multicolumn{4}{|l|}{$\begin{array}{l}\text { Estoy de acuerdo en la forma en que mi } \\
\text { familia expresa afecto y responde a mis sen- } \\
\text { timientos (angustia, amor y pena) }\end{array}$} \\
\hline $\begin{array}{l}\text { Estoy satisfecho con la cantidad de tiempo } \\
\text { que mi familia y yo pasamos juntos }\end{array}$ & & & \\
\hline
\end{tabular}

el puntaje en la EDE y el nivel educacional, tipo de previsión, actividad laboral, percepción de funcionalidad familiar, medida a través del APGAR familiar (Tabla 5), hacinamiento, promiscuidad, número de personas que viven en la misma casa y características de vivienda entre otras. El análisis no paramétrico de las variables realizado a través del coeficiente de Spearman mostró una correlación significativa sólo entre el puntaje de la EDE y el puntaje del APGAR familiar $(r=-0,38$ y $\mathrm{p}<0,01)$ y entre el puntaje de la EDE y el hacinamiento $(r=0,22$ y $p<0,01)$. Asimismo, el hacinamiento estuvo correlacionado con el puntaje de APGAR familiar, es decir, en la medida que el hacinamiento aumentó disminuyó la percepción de la funcionalidad familiar.

Por otro lado, entre las variables obstétricas se encontró una correlación directa y significativa entre el número de partos y el puntaje en la EDE $(\mathrm{r}=0,21 ; \mathrm{p}<0,05)$.

Finalmente, se ajustó un modelo de regresión múltiple para determinar si estas variables hacen un aporte significativo en forma conjunta a la explicación del puntaje de la Escala de Edimburgo.

Los resultados mostraron que aunque las 3 variables están asociadas en forma significativa con depresión postparto en forma univariada, al ajustar un modelo de regresión múltiple se observa que, en presencia de APGAR familiar, ninguna de las otras dos variables hace un aporte significativo para explicar la sintomatología depresiva.

\section{Discusión}

El nacimiento del primer hijo marca un cambio en la vida de la mujer, la que deberá trabajar para incorporar al nuevo ser que llega basándose en sus experiencias previas y venciendo el temor de perder la identidad lograda en etapas previas ${ }^{17}$. En ese sentido el proceso del parto representa una de las experiencias más paradójicas que vive la mujer ya que, si bien está permitiendo la expresión de la vida, también debe enfrentarse a una experiencia psicosocial profunda, que pone a prueba su feminidad y sus competencias personales ${ }^{18}$. Relacionado con lo anterior, se estima que $80 \%$ de las mujeres presentan fluctuaciones en su ánimo durante el puerperio. Entre ellos, la depresión posparto que es un trastorno de la salud mental de gran relevancia y a la cual se le han atribuido variados factores de riesgo. En Chile, se estima que su prevalencia en población general es de $27,7 \%$ a $41,3 \%{ }^{5}$, lo que sitúa el $37,1 \%$ obtenido en esta investigación dentro de un rango esperado. Sin embargo, si se considera que se estudió una muestra de mujeres de bajo riesgo, la importancia de esta cifra cambia radicalmente. La sintomatología depresiva del puerperio en general, se ha asociado fuertemente con los antecedentes de cuadros ansiosos y depresivos previos, con la morbilidad obstétrica y perinatal y con variables como el tipo de parto, la paridad, el nivel educacional y el estado civil entre otras. 
En este estudio el nivel alto de escolaridad podría estar actuando como factor protector frente al cuadro depresivo, al igual que la posible influencia del parto vaginal, asociándolo a un "deber cumplido" desde el rol social asignado a la maternidad, sin embargo, ambas situaciones requieren de mayor investigación.

Los resultados de este estudio muestran que la percepción materna de funcionalidad familiar es la única variable que explica en parte la ocurrencia de sintomatología depresiva en puérperas de bajo riesgo obstétrico, lo que se relaciona con hallazgos de otras investigaciones que señalan que el no contar con el apoyo de la familia constituye un factor significativo en el riesgo para el desarrollo de cuadros depresivos en el puerperio ${ }^{2,19,20}$. Aparentemente, es posible suponer que las relaciones familiares tendrían mayor repercusión en la afectividad de la mujer, en especial en períodos de mayor sensibilidad. La adaptación fisiológica junto con las tareas propias de la incorporación del hijo(a) a la vida diaria, producen tensiones que colocan a la mujer en una situación de vulnerabilidad que favorecería la aparición de sintomatología depresiva. Incorporar elementos de evaluación temprana de la funcionalidad familiar pudiera contribuir en el diseño de intervenciones precoces que impacten sobre la salud materna. El APGAR familiar es un instrumento auto-administrado de tamizaje, fácil de aplicar y de interpretar, que consta de cinco preguntas cerradas de carácter general para medir el grado de satisfacción con que el encuestado percibe la funcionalidad de su familia. Si bien su resultado no mide la disfunción familiar en sí misma, existe buena correlación entre puntajes bajos y la presencia de problemas relacionales ${ }^{21}$.

Por último, se debe tener en cuenta que los síntomas anímicos muchas veces se confunden con las características de este período adaptativo. Si se asocia esta situación a la condición de bajo riesgo materno, es posible que la mujer tenga menos posibilidades de ser pesquisada a tiempo durante sus controles postnatales.

Una herramienta eficiente y validada en Chile, para la pesquisa de este cuadro es la Escala de Depresión de Edimburgo. La simplicidad de este instrumento permite que cualquier profesional de la salud lo utilice durante la atención de salud, derivando a control médico para precisar el diagnóstico, sólo en aquellos casos en que se sospeche efectivamente la presencia de un cuadro depresivo.
La política pública vigente recomienda su uso en la octava semana del post parto, período en que ha disminuido la sintomatología adaptativa normal. El diagnóstico precoz y la instauración de un plan terapéutico de abordaje eficiente, adecuado y a tiempo, repercutirá directamente en la calidad de vida de las madres, sus hijos(a) y sus familias; disminuirá las complicaciones a corto y largo plazo e influirá en la disminución de los costos en salud. El tratamiento de la depresión está considerado en el Régimen de Garantías Explicitas en Salud al incorporar intervenciones psicosociales individuales y grupales, además del tratamiento farmacológico, condición que permite diseñar estrategias de intervención temprana sobre aquellos factores de riesgo que puedan ser modificables, lo que a su vez, contribuye a mejorar el capital social de las familias chilenas.

\section{Referencias}

1. Dennis C, McQueen K. The relationship between infantfeeding outcomes and postpartum depression: A qualitative systematic review. Pediatrics 2009; 123: 736-51.

2. Rojas G, Fritsh R, Solís J, González M, Guajardo V, Araya R. Calidad de vida de mujeres deprimidas en el posparto. Rev Med Chile 2006; 134: 713-20.

3. National Health and Medical Research Council. Postnatal depression. A systematic review of published scientific literature to 1999. Canberra Australia: National Health and Medical Research Council; 2000.

4. Yim I, Glynn L, Dunkel-Schetter C, Hobel C, ChiczDeMet A, Sandman C. Risk of postpartum depressive symptoms with elevated corticotropin-releasing hormone in human pregnancy. Arch Gen Psychiatry 2009; 66: 162-69.

5. Ministerio de Salud. Guía clínica: Tratamiento de personas con depresión. Santiago de Chile: Ministerio de Salud; 2006.

6. Field T. Postpartum depression effects on early interactions, parenting, and safety practices: A review. Infant Behav Dev 2010; 33: 1-6.

7. Castañón C, Pinto J. Mejorando la pesquisa de depresión posparto a través de un instrumento de tamizaje, la escala de depresión posparto de Edimburgo. Rev Med Chile 2008; 136: 851-8.

8. Dennis CL, Hodnett E. Intervenciones psicosociales $y$ psicológicas para el tratamiento de la depresión postparto. Chichester, John Wiley \& Sons, 2008

9. Rojas G, Fritsch R, Guajardo V, Rojas F, Barroilhet S, 
Jadresic E. Caracterización de madres deprimidas en el posparto. Rev Med Chile 2010; 138: 536-42.

10. Hamdan A, Tamim H. Psychosocial risk and protective factors for postpartum depression in the United Arab Emirates. Arch Womens Ment Health 2011; 14: 125-33. Disponible en http://www.springerlink.com/content/ g3x2tr14l14w23g1/ [consultado el 8 de julio de 2011].

11. McCoy S, Beal J, Shipman S, Payton M, Watson G. Risk factors for postpartum depression: A retrospective investigation at 4-weeks postnatal and a review of the literature. J Am Osteopath Assoc 2006; 106: 193-98.

12. Marín D, Bullones A, Carmona F, Carretero MI, Moreno MA, Peñacoba C. Influencia de los factores psicológicos en el embarazo, parto y puerperio. Un estudio longitudinal. Nure Investigación 2008; 37 Disponible en http://www.fuden.eu/FICHEROS_ADMINISTRADOR/ INV_NURE/proyemb37210200893951.pdf [consultado el 4 de diciembre de 2011].

13. Paulson J, Bazemore S. Prenatal and postpartum depression in fathers and its association with maternal depression: A meta-analysis. JAMA 2010; 303: 1961-9.

14. Beck C. Predictors of postpartum depression: an update. Nurs Res. 2001; 50: 275-85.

15. Jadresic E, Araya R, Jara C. Validation of the Edinburgh Postnatal Depression Escale (EDE) in Chilean postpar- tum women. J Psychosom Obstet Gynaecol. 1995; 16: 187-91.

16. Maroto G, García M, Fernández A. Evaluación del estado del ánimo en el puerperio con la Escala de Depresión Postnatal de Edimburgo. Int J Clin Health Psychol 2004; 5: 305-18.

17. Florenzano R. En el Camino de la Vida. Chile: Editorial Universitaria, 1993.

18. Callister L, Khalaf I, Semenic S, Kartchner R, Vehvilainen-Julkunen $\mathrm{K}$. The pain perception of culturally diverse women. Pain Management Nursing 2003; 4 (4): 145-54.

19. Urdaneta J, Rivera A, García J, Guerra M, Baabel N, Contreras A. Factores de riesgo de depresión posparto en puérperas venezolanas valoradas por medio de la escala de Edimburgo. Rev Chil Obstet Ginecol 2011; 76: 102-12.

20. Alvarado C, Cifuentes A, Estrada S, Salas C, Hernández A, Ortiz S, et al. Prevalencia de depresión posnatal en mujeres atendidas en hospitales públicos de Durango, México. Gac Med Mex 2010; 146: 1-9.

21. Maddaleno M, Horwitz N, Jara C, Florenzano R, Salazar D. Aplicación de un instrumento para calificar el funcionamiento familiar en la atención de adolescentes. Rev Chil Pediatr 1987; 58: 246-49. 